\title{
Frequency-dependent tidal dissipation in a viscoelastic Saturnian core and expansion of Mimas' semi-major axis
}

\author{
D. Shoji and H. Hussmann
}

\begin{abstract}
German Aerospace Center(DLR), Institute of Planetary Research, Rutherfordstr. 2, 12489 Berlin, Germany e-mail: [Daigo.Shoji; Hauke.Hussmann]@dlr.de
\end{abstract}

Received 12 December 2016 / Accepted 13 February 2017

\begin{abstract}
Context. Regarding tidal dissipation in Saturn, usually parameterized by Saturn's quality factor $Q$, there remains a discrepancy between conventional estimates and the latest determination that has been derived from astrometric observations of Saturn's inner satellites. If dissipation in Saturn is as large as the astrometric observations suggest, and is independent of time and tidal frequency, conventional models predict that Mimas' initial orbit should be located inside Saturn's synchronous orbit or even inside its Roche limit, in contradiction to formation models.

Aims. Using simple structure models and assuming Saturn's core to be viscoelastic, we look for dissipation models which are consistent with both the latest astrometric observations and with Mimas' orbital migration.

Methods. Firstly, using a two-layer model of Saturn's interior structure, we constrain the ranges of rigidity and viscosity of Saturn's core which are consistent with Saturn's dissipation derived from astrometric observations at the tidal frequencies of Enceladus, Tethys, and Dione. Next, within the constrained viscosity and rigidity ranges, we calculate Mimas' semi-major axis considering the frequency dependence of viscoelastic dissipation in Saturn's core. By the two calculations, we evaluate (1) Saturnian models which can explain the astrometrically determined Saturnian dissipation, and (2) whether Mimas' initial semi-major axis is larger than the synchronous orbit.

Results. We show that if the core is assumed to be solid with a viscosity of $10^{13}-10^{14} \mathrm{~Pa}$ s (depending on its size), the lower boundary of the observed Saturnian dissipation at tidal frequencies of Enceladus, Tethys, and Dione $\left(k_{2 \mathrm{~s}} / Q_{\mathrm{s}} \sim 4 \times 10^{-5}\right.$ where $k_{2 \mathrm{~s}}$ is Saturn's second degree Love number and $Q_{\mathrm{s}}$ its quality factor) can be explained by our model. In this viscosity range, Mimas can stay outside the synchronous orbit and the Roche limit for 4.5 billion years of evolution.

Conclusions. In the case of a frequency dependent viscoelastic dissipative core, the lower boundary of the observed Saturnian dissipation can be consistent with the orbital expansion of Mimas. In this model, the assumption of a late formation of Mimas, discussed recently, is not required.
\end{abstract}

Key words. planets and satellites: interiors - planets and satellites: individual: Saturn - planets and satellites: individual: Mimas

\section{Introduction}

Tidal dissipation in Saturn induced by its moons can be one important factor constraining the interior structure and dynamics of Saturn. The magnitude of dissipation is estimated by the quality factor, $Q$, which is defined as the ratio between the peak of the stored energy and the dissipated energy over one tidal cycle. A small $Q$ value means that a large quantity of energy is dissipated in Saturn.

Although the Saturnian $Q$ value is not constrained well, its minimum value has been evaluated by the orbital expansion of Mimas (e.g., Goldreich \& Soter 1966; Gavrilov \& Zharkov 1977; Murray \& Dermott 2000). The tidal bulge on Saturn exerted by Mimas is ahead of the Saturn-Mimas axis because Saturn's rotation period is smaller than the orbital period of Mimas. This tidal bulge adds a torque to Mimas and the semi-major axis of Mimas increases. Ignoring dissipation in Mimas, the increasing rate of semi-major axis $a$ of Mimas is given (e.g., Murray \& Dermott 2000) by

$\frac{\mathrm{d} a}{\mathrm{~d} t}=3 \sqrt{\frac{G}{M_{\mathrm{s}}}} \frac{M_{\mathrm{m}} R_{\mathrm{s}}^{5}}{a^{5.5}} \frac{k_{2 \mathrm{~s}}}{Q_{\mathrm{s}}}$, where $t, G, M_{\mathrm{s}}, M_{\mathrm{m}}$, and $R_{\mathrm{s}}$ are time, the gravitational constant, Saturnian mass, Mimas' mass, and Saturnian radius, respectively. The parameters $k_{2 \mathrm{~s}}$ and $Q_{\mathrm{s}}$ are the second degree Love number and the $Q$ value of Saturn. By integrating Eq. (1) backwards in time, the past semi-major axis of Mimas can be calculated. As this equation shows, the change of the orbit becomes large when $Q_{\mathrm{s}}$ is small. In order to move to the current orbit from a small initial semi-major axis in the past, Mimas must be located outside of the surface (and the Roche limit) of Saturn when it was formed. One of the simplest assumptions for the evaluation of $Q_{\mathrm{s}}$ is that it is constant along the evolution of the planet and that it is independent of the forcing frequency (e.g., MacDonald 1964; Kaula 1964). The tidal $Q$ of Saturn had so far been constrained by considering that Mimas took at least 4.5 billion years to move from a quasisynchronous orbit with Saturn to its present location, yielding $Q_{\text {s }}>18000$ (e.g., Goldreich \& Soter 1966; Gavrilov \& Zharkov 1977; Murray \& Dermott 2000; Meyer \& Wisdom 2007). However, recent astrometric measurements of Saturnian satellites have indicated that the $Q$ value of Saturn is much lower (Lainey et al. 2012, 2017). The studies reported above discuss the Saturnian dissipation by the value of $Q_{\mathrm{s}}$ assuming the value of $k_{2 s}$ derived by Gavrilov \& Zharkov (1977). However, from 
the astrometric measurements the value of $k_{2 \mathrm{~s}} / Q_{\mathrm{s}}$ can be obtained rather than $Q_{\mathrm{s}}$. Lainey et al. (2017) show that $k_{2 \mathrm{~s}} / Q_{\mathrm{s}}$ of Saturn is around $10^{-4}$ (the detailed values are shown below), which implies that $Q_{\mathrm{s}}$ is a few thousand only, even if we consider the latest determination of $k_{2 \mathrm{~s}}=0.413$ (Wahl et al. 2017). As a mechanism of the observed dissipation of Saturn, Lainey et al. (2017) suggest a viscoelastic response of the solid core. Although a viscoelastic core can generate significant dissipation (Remus et al. 2012, 2015; Lainey et al. 2017), it contradicts the conventional estimations derived from the orbital expansion of Mimas (e.g., Goldreich \& Soter 1966; Gavrilov \& Zharkov 1977; Murray \& Dermott 2000; Meyer \& Wisdom 2007). One hypothesis is that the Saturnian satellites such as Mimas were formed recently (Charnoz et al. 2011). If Mimas moved to the current position on a small timescale, strong constant dissipation inside Saturn does not contradict the orbital expansion of Mimas.

Dissipation of Saturn plays an important role for the possible activity of the Saturnian satellites. The Cassini probe has observed that Enceladus is a currently active body emanating water plumes, possibly implying liquid water in its subsurface (e.g., Porco et al. 2006). If Enceladus is in an equilibrium state of the resonance with Dione, the tidal heating rate generated in Enceladus is $\sim 1.1 \mathrm{GW}$ at $Q_{\mathrm{s}}=18000$ (Meyer \& Wisdom 2007), which is independent of the interior structure of Enceladus. However, the equilibrium heating rate increases with decreasing $Q_{\mathrm{S}}$ (Meyer \& Wisdom 2007; Lainey et al. 2012), which may affect Enceladus' structure and activity.

In this work, although the detailed structure and the state of the Saturnian core (e.g., whether the core is solid or liquid) is uncertain, we reconsider the conventional estimations derived from the orbital migration of Mimas assuming that Saturn's core is solid and viscoelastic. In case of a viscoelastic response, both Love number and $Q$ value depend on the frequency of the cyclic forcing. Because the tidal frequency on Saturn changes with Mimas' semi-major axis, $k_{2 \mathrm{~s}}$ and $Q_{\mathrm{s}}$ in Eq. (1) cannot be constant. It has been demonstrated that constant $Q$ is a strong assumption and frequency dependent $Q$ can lead to different tidal rotational and orbital evolution (e.g., Efroimsky \& Lainey 2007; Auclair-Desrotour et al. 2014). We calculate the past semi-major axis of Mimas taking into account the frequency dependence of the dissipation with the simple two-layer structure models by Remus et al. (2012, 2015). Firstly we constrain the rigidity and viscosity of the solid core, which are consistent with the latest observational results by Lainey et al. (2017), and then we calculate the semi-major axis within the range of the constrained rheological parameters. Here, we consider the value of $k_{2 \mathrm{~s}} / Q_{\mathrm{s}}$ rather than $Q_{\mathrm{s}}$ because $k_{2 \mathrm{~s}} / Q_{\mathrm{s}}$ is the directly obtained parameter by astrometric methods. By the two calculations, we suggest the Saturnian models which can be consistent with both Mimas' orbital evolution and the latest observational results of Saturnian dissipation.

\section{Structure and rheology of Saturn}

Remus et al. (2012, 2015) have explained the large dissipation of Saturn using the two layer model with fluid envelope and viscoelastic solid core. Here we assume this simple structure model because the purpose of this work is to relate the observed dissipation to Mimas' orbital change. Mass and size of Saturn are shown in Table 1, which are consistent with Remus et al. (2012, 2015). Lainey et al. (2017) assume more detailed models with layered structure and the calculation method by Tobie et al. (2005), and mention that the viscosity range to generate the observed $Q_{\mathrm{s}}$ is
Table 1. Physical parameters and values.

\begin{tabular}{lccc}
\hline \hline Parameter & Symbol & Value & Unit \\
\hline Radius of Earth & $R_{\oplus}$ & $6.371 \times 10^{6}$ & $\mathrm{~m}$ \\
Mass of Earth & $M_{\oplus}$ & $5.9736 \times 10^{24}$ & $\mathrm{~kg}$ \\
Radius of Saturn & $R_{\mathrm{s}}$ & $9.14 R_{\oplus}$ & \\
Mass of Saturn & $M_{\mathrm{s}}$ & $95.159 M_{\oplus}$ & \\
Mass of solid core & $M_{\mathrm{c}}$ & $18.65 M_{\oplus}$ & \\
Rotation rate of Saturn & $\Omega$ & $1.65 \times 10^{-4}$ & $\mathrm{rad} \mathrm{s}^{-1}$ \\
Mass of Mimas & $M_{\mathrm{m}}$ & $3.7493 \times 10^{19}$ & $\mathrm{~kg}$ \\
Current $a$ of Mimas & $a_{0}$ & $1.8552 \times 10^{8}$ & $\mathrm{~m}$ \\
\hline
\end{tabular}

compatible between their complex model and the simple two layer model. In the structure models by Remus et al. (2012, 2015) and Lainey et al. (2017), the fluid envelope is assumed to be non-dissipative. Convective turbulent friction applied on fluid tidal waves (e.g., Ogilvie \& Lin 2004) and the resonant-lock mechanism (Fuller et al. 2016) may contribute to the dissipation in Saturn, which can compete with the viscoelastic dissipation in the solid core (Guenel et al. 2014). Here we consider dissipation only in the solid core as an end-member model. The effect of dissipation in the envelope will be addressed in future studies. In the case of viscoelastic material, due to the delay of the response to the forcing because of viscous friction, the Love number becomes complex $\tilde{k}_{2 \mathrm{~s}}$, which is given (Remus et al. 2015) by

$\tilde{k}_{2 \mathrm{~s}}=\frac{3}{2} \frac{\tilde{\epsilon}+\frac{2}{3} \beta}{\alpha \tilde{\epsilon}-\beta}$,

where

$\alpha=1+\frac{5}{2}\left(\frac{\rho_{\mathrm{c}}}{\rho_{\mathrm{o}}}-1\right)\left(\frac{R_{\mathrm{c}}}{R_{\mathrm{s}}}\right)^{3}$

$\beta=\frac{3}{5}\left(\frac{R_{\mathrm{c}}}{R_{\mathrm{S}}}\right)^{2}(\alpha-1)$

$\tilde{\epsilon}=\frac{\frac{19 \tilde{\mu}_{\mathrm{c}}}{2 \rho_{\mathrm{c}} g_{\mathrm{c}} R_{\mathrm{c}}}+\frac{\rho_{\mathrm{o}}}{\rho_{\mathrm{c}}}\left(1-\frac{\rho_{\mathrm{o}}}{\rho_{\mathrm{c}}}\right)\left(\beta+\frac{3}{2}\right)+\left(1-\frac{\rho_{\mathrm{o}}}{\rho_{\mathrm{c}}}\right)}{\left(\alpha+\frac{3}{2}\right) \frac{\rho_{\mathrm{o}}}{\rho_{\mathrm{c}}}\left(1-\frac{\rho_{\mathrm{o}}}{\rho_{\mathrm{c}}}\right)}$.

The parameters $R_{\mathrm{c}}, g_{\mathrm{c}}, \rho_{\mathrm{c}}$, and $\rho_{\mathrm{o}}$ are radius of the core, gravitational acceleration at the surface of the core, density of the core, and density of the envelope, respectively. We consider the value of $R_{\mathrm{c}}$ between $0.2 R_{\mathrm{s}}$ and $0.24 R_{\mathrm{s}}$, which includes the core radius by Remus et al. (2015). $g_{\mathrm{c}}, \rho_{\mathrm{c}}$, and $\rho_{\mathrm{o}}$ can be calculated from the mass of Saturn $M_{\mathrm{s}}$, the mass of the core $M_{\mathrm{c}}, R_{\mathrm{S}}$ and $R_{\mathrm{c}}$ shown in Table $1 . \tilde{\mu}_{\mathrm{c}}$ is the complex shear modulus of the viscoelastic core. In this work, we assume a Maxwell rheology in which the complex shear modulus of the Maxwell model is given by

$\tilde{\mu}_{\mathrm{c}}=\frac{\omega_{\mathrm{f}} \mu \eta}{\omega_{\mathrm{f}} \eta+i \mu}$,

where $\mathrm{i}=\sqrt{-1}$. The parameters $\mu, \eta$ and $\omega$ are the tidally effective rigidity and viscosity of the solid core of Saturn and the forcing frequency, respectively. For the frequency of tides on Saturn by satellites, $\omega_{\mathrm{f}}$ can be represented by the rotational angular velocity of Saturn $\Omega$ and the mean motion of the satellites $\omega$ as $\omega_{\mathrm{f}}=2(\Omega-\omega)$. Because $Q_{\mathrm{s}}=\left|\tilde{k}_{2 \mathrm{~s}}\right| / \tilde{I}_{\tilde{k}}\left(\tilde{k}_{2 \mathrm{~s}}\right) \mid$ (e.g., Remus et al. 2012), $k_{2 \mathrm{~s}} / Q_{\mathrm{s}}=\left|\operatorname{Im}\left(\tilde{k}_{2 \mathrm{~s}}\right)\right|$ with $k_{2 \mathrm{~s}}=\left|\tilde{k}_{2 \mathrm{~s}}\right|$. 
D. Shoji and H. Hussmann: Saturnian dissipation by frequency dependent core
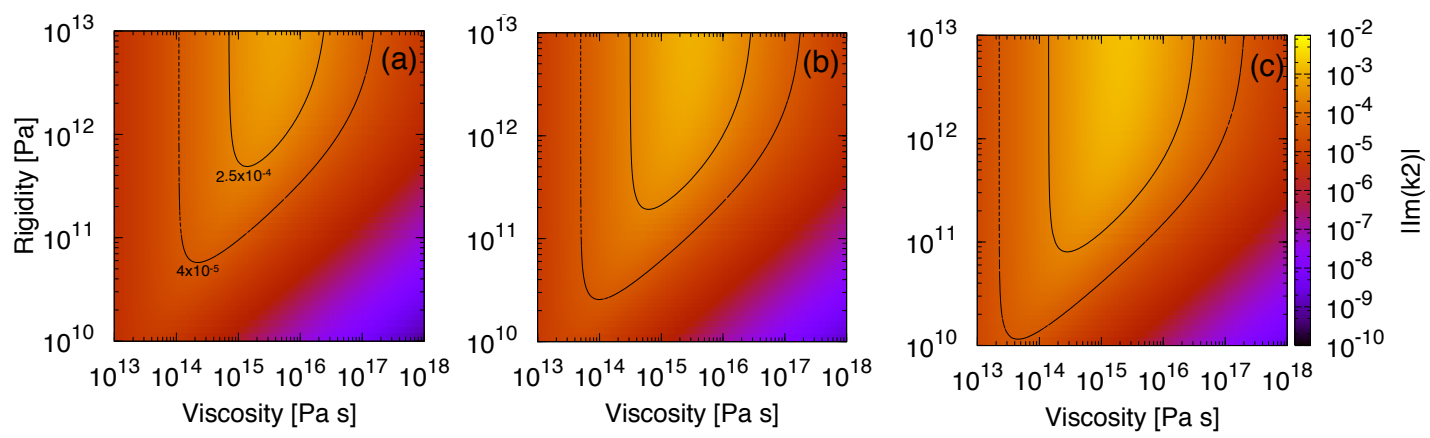

Fig. 1. $\left|\operatorname{Im}\left(\tilde{k}_{2 \mathrm{~s}}\right)\right|$ as a function of viscosity and rigidity of the solid core. $\omega_{\mathrm{f}}=2.5 \times 10^{-4} \mathrm{rad} \mathrm{s}^{-1}$ and the core radius is a) $0.2 R_{\mathrm{s}}$ b) $0.219 R_{\mathrm{s}}$; and c) $0.24 R_{\mathrm{s}}$. Two contours show the upper $\left|\operatorname{Im}\left(\tilde{k}_{2 \mathrm{~s}}\right)\right|=2.5 \times 10^{-4}$ and the lower $\left|\operatorname{Im}\left(\tilde{k}_{2 \mathrm{~s}}\right)\right|=4 \times 10^{-5}$ boundaries which are consistent with the observed values.
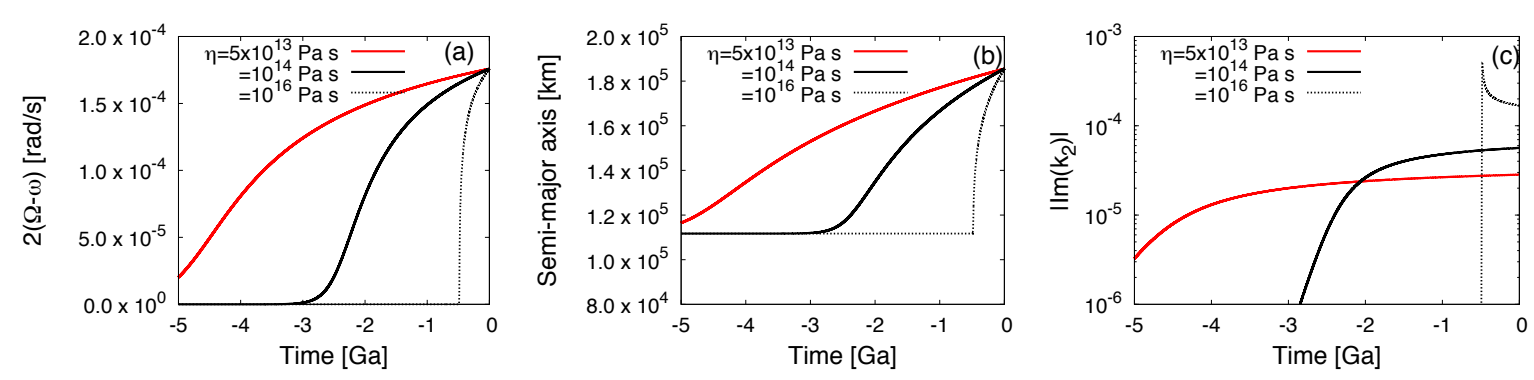

Fig. 2. Evolutions of a) $2(\Omega-\omega)$ b) $\left|\operatorname{Im}\left(\tilde{k}_{2 \mathrm{~s}}\right)\right|$ and c) $a$ with different $\eta$ at $R_{\mathrm{c}}=0.219 R_{\mathrm{s}}$ and $\mu=5 \times 10^{11} \mathrm{~Pa}$. Integrations are conducted backwards in time and the current $a$ of Mimas is shown at $t=0$.

\section{Method}

\subsection{Constraint of rigidity and viscosity for observed dissipation}

Firstly we calculate the ranges of rigidity and viscosity which are consistent with the observed $k_{2 \mathrm{~s}} / Q_{\mathrm{s}}$ at the tidal frequencies caused by Enceladus, Tethys, and Dione. Lainey et al. (2017) also estimated $k_{2 \mathrm{~s}} / Q_{\mathrm{s}}$ at Rhea's frequency. However, they suggest that the dissipation at Rhea's frequency is caused by turbulent friction in the envelope, which is beyond the scope of this work. The value of $k_{2 \mathrm{~s}} / Q_{\mathrm{s}}$ is $(20.70 \pm 19.91) \times 10^{-5}$, $(15.84 \pm 12.26) \times 10^{-5}$ and $(16.02 \pm 12.72) \times 10^{-5}$ at the tidal frequencies of Enceladus, Tethys, and Dione, respectively, and $(1.59 \pm 0.74) \times 10^{-4}$ in a global estimation (Lainey et al. 2017). Thus, we consider $\left|\operatorname{Im}\left(\tilde{k}_{2 \mathrm{~s}}\right)\right|$ between $4 \times 10^{-5}$ and $2.5 \times 10^{-4}$. $k_{2 \mathrm{~s}} / Q_{\mathrm{s}}$ is almost independent of the mean motion difference among these three satellites (Lainey et al. 2017). We set $\omega_{\mathrm{f}}=$ $2.5 \times 10^{-4} \mathrm{rad} \mathrm{s}^{-1}$, which is between the tidal frequencies of Enceladus and Dione.

\subsection{Change of Mimas' semi-major axis}

As a next step, we calculate the initial semi-major axis of Mimas with the constrained rigidity and viscosity. Assuming a Kepler orbit, the mean motion of Mimas is given by $\omega=\sqrt{G M_{\mathrm{s}} / a^{3}}$. Substituting $\left|\operatorname{Im}\left(\tilde{k}_{2 \mathrm{~s}}\right)\right|$ in Eq. (1), $\mathrm{d} a / \mathrm{d} t$ and dissipation of Saturn can be coupled by Eqs. (1)-(6). Integrations of $a$ are performed backwards from the current semi-major axis $a_{0}=1.8552 \times$ $10^{8} \mathrm{~m}$ (Murray \& Dermott 2000) with a Runge-Kutta method and $10^{4} \mathrm{yr}$ of time step. We performed additional calculations with time steps of $10^{3} \mathrm{yr}$ and $5 \times 10^{2} \mathrm{yr}$ and the results did not change. For simplicity, the angular velocity of Saturn's rotation and the mass of Mimas are fixed at $\Omega=1.65 \times 10^{-4} \mathrm{rad} \mathrm{s}^{-1}$ (Giampieri et al. 2006; Anderson \& Schubert 2007) and $M_{\mathrm{m}}=$ $3.7493 \times 10^{19} \mathrm{~kg}$ (Jacobson et al. 2006), respectively.

\section{Results}

\subsection{Rigidity and viscosity ranges for observed dissipation}

Figure 1 shows $\left|\operatorname{Im}\left(\tilde{k}_{2 \mathrm{~s}}\right)\right|$ as a function of rigidity and viscosity of Saturn's core. The rigidity and viscosity ranges which are consistent with the observed Saturnian dissipation (Fig. 2) are compatible with the rheological values estimated by Remus et al. (2015). $\left|\operatorname{Im}\left(\tilde{k}_{2 \mathrm{~s}}\right)\right|$ becomes maximum at around $10^{15} \mathrm{~Pa}$ s. Although rigidity and viscosity which attain the observed $\left|\operatorname{Im}\left(\tilde{k}_{2 \mathrm{~s}}\right)\right|$ change with the core radius, minimum viscosities for the observed dissipation should be $10^{13}-10^{14} \mathrm{~Pa}$. If $\eta \sim 10^{15} \mathrm{~Pa} \mathrm{~s},\left|\operatorname{Im}\left(\tilde{k}_{2 \mathrm{~s}}\right)\right|$ becomes too high to be consistent with the observed values.

\subsection{Evolutions of dissipation and Mimas' orbit}

Figure 2 shows the evolutions of tidal frequency $2(\Omega-\omega)$, $\left|\operatorname{Im}\left(\tilde{k}_{2 \mathrm{~s}}\right)\right|$, and semi-major axis $a$ at $R_{\mathrm{c}}=0.219 R_{\mathrm{s}}$. The rigidity $\mu$ is $5 \times 10^{11} \mathrm{~Pa}$ and the viscosity $\eta=5 \times 10^{13}, 10^{14}$ and $10^{16} \mathrm{Pas}$, which are consistent with the reasonable parameter range (Remus et al. 2015) and the observed $k_{2 \mathrm{~s}} / Q_{\mathrm{s}}$ at the frequency of Enceladus, Tethys, and Dione (Fig. 1). Due to the dissipation of Saturn, the semi-major axis decreases with decreasing time (Mimas' orbit expands with time). Because we assume $\omega=\sqrt{G M_{\mathrm{s}} / a^{3}}, 2(\Omega-\omega)$ also decreases as time decreases. However, once the semi-major axis decreases to the synchronous orbit with Saturn in which Mimas' orbital period is the same as the rotational period of Saturn $(\Omega=\omega)$, dissipation does not occur because $2(\Omega-\omega)$ becomes zero, and thus the migration of Mimas stops. At $\eta=5 \times 10^{13} \mathrm{~Pa} \mathrm{~s}$ and $10^{14} \mathrm{~Pa} \mathrm{~s},\left|\operatorname{Im}\left(\tilde{k}_{2 \mathrm{~s}}\right)\right|$ decreases with decreasing time (Fig. 2b). In this viscosity range, $2(\Omega-\omega) / 2 \pi$ is smaller than the Maxwell frequency $(\mu / \eta)$. Thus the response of the solid core is like a fluid as $a$ decreases $(2(\Omega-\omega)$ decreases) and the core becomes less dissipative. On the other hand, at $\eta=10^{16} \mathrm{~Pa} \mathrm{~s},\left|\operatorname{Im}\left(\tilde{k}_{2 \mathrm{~s}}\right)\right|$ increases with decreasing time because 


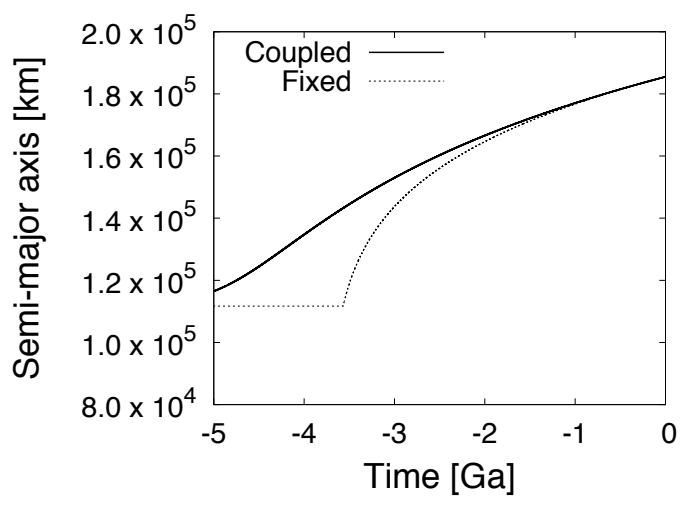

Fig. 3. Comparison of semi-major axis between constant $\left|\operatorname{Im}\left(\tilde{k}_{2 \mathrm{~s}}\right)\right|$ at $2.8 \times 10^{-5}$ and coupled orbital $\left|\operatorname{Im}\left(\tilde{k}_{2 \mathrm{~s}}\right)\right|$ at $R_{\mathrm{c}}=0.219 R_{\mathrm{s}}$ and $\mu=5 \times$ $10^{11} \mathrm{~Pa}$. Once Mimas gets into the synchronous orbit, we assume that $a$ does not change.

$2(\Omega-\omega) / 2 \pi$ at $t=0$ is larger than the Maxwell frequency. By the decrease of $2(\Omega-\omega)$ (increase of $\omega)$, the response of the solid core becomes viscoelastic from elastic, which results in large dissipation. Due to the large dissipation, $a$ gets into the synchronous orbit at around $-0.5 \mathrm{Ga}$ (Fig. 2c).

One important result is that, at $\eta=5 \times 10^{13} \mathrm{~Pa} \mathrm{~s}, 2(\Omega-\omega)$ does not become zero and thus $a$ does not get into the synchronous orbit at the time of solar system formation (around $-4.5 \mathrm{Ga}$ ). Thus, if the viscosity of Saturn is around $\eta=5 \times$ $10^{13} \mathrm{Pas}$, the latest observational dissipation at the frequency of Enceladus, Tethys, and Dione does not contradict the conventional evaluations that Mimas must have been outside of the surface of Saturn and the synchronous orbit 4.5 billion years ago (e.g., Goldreich \& Soter 1966; Gavrilov \& Zharkov 1977; Murray \& Dermott 2000; Meyer \& Wisdom 2007). In addition to the synchronous orbit, Mimas should be outside of the Roche limit. Assuming that Mimas is a rigid and spherical body, the distance of the Roche limit $a_{\mathrm{L}}=R_{\mathrm{s}}\left(3 \rho_{\mathrm{s}} / \rho_{\mathrm{m}}\right)^{1 / 3}$ (Murray \& Dermott 2000), while $a_{L}=2.456 R_{\mathrm{s}}\left(\rho_{\mathrm{s}} / \rho_{\mathrm{m}}\right)^{1 / 3}$ if Mimas is fluid (Chandrasekhar 1969), where $\rho_{\mathrm{s}}$ and $\rho_{\mathrm{m}}$ are the density of Saturn and Mimas, respectively. Using the values of our model $\left(\rho_{\mathrm{s}}=687.3 \mathrm{~kg} \mathrm{~m}^{-3}\right)$ and the mean density of Mimas at $\rho_{\mathrm{m}}=1150 \mathrm{~kg} \mathrm{~m}^{-3}$ (Thomas et al. 2007), $a_{\mathrm{L}}$ is $\sim 7 \times 10^{4} \mathrm{~km}$ for the rigid Mimas and $\sim 1.2 \times 10^{5} \mathrm{~km}$ in the case of the fluid Mimas, respectively. Even if Mimas is a fluid body, it stays outside of the Roche limit at $\eta=5 \times 10^{13} \mathrm{~Pa}$ s (Fig. 2).

We calculated Mimas' orbit with different rigidity within the consistent range to the observations. Although the magnitude of dissipation depends on rigidity, the value of viscosity strongly affects whether Mimas gets into the synchronous orbit because $\left|\operatorname{Im}\left(\tilde{k}_{2 \mathrm{~s}}\right)\right|$ is relatively independent of rigidity at $\eta \sim 10^{13}-10^{14} \mathrm{Pas}$ (Fig. 1). Thus, as long as the rigidity is consistent with the observations (Fig. 1), the conclusion that Mimas does not get into the synchronous orbit at the lower boundary of the viscosity does not change.

In addition to low viscosity, the frequency dependence of $\left|\operatorname{Im}\left(\tilde{k}_{2 \mathrm{~s}}\right)\right|$ is another reason why Mimas does not get into the synchronous orbit and the Roche limit. In the case of $\eta=$ $5 \times 10^{13} \mathrm{~Pa} \mathrm{~s},\left|\operatorname{Im}\left(\tilde{k}_{2 \mathrm{~s}}\right)\right|$ is approximately $2.8 \times 10^{-5}$ at $t=0$ (Fig. 2b). Figure 3 compares $a$ of the case in which $\left|\operatorname{Im}\left(\tilde{k}_{2 \mathrm{~s}}\right)\right|$ is fixed at $2.8 \times 10^{-5}$ with the frequency dependent $\left|\operatorname{Im}\left(\tilde{k}_{2 \mathrm{~s}}\right)\right|$ (coupled model of dissipation and orbit). While the semi-major axis is larger than the synchronous orbit for 4.5 billion years in the coupled model, Mimas' orbit becomes synchronous with Saturn
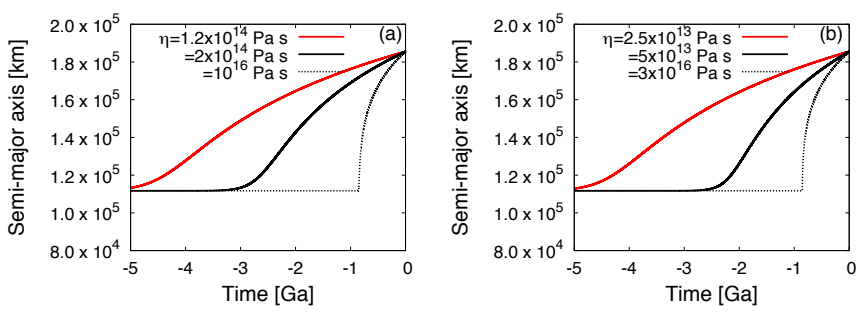

Fig. 4. Evolutions of semi-major axis at a): $R_{\mathrm{c}}=0.2 R_{\mathrm{s}}$ and $\left.\mathbf{b}\right): R_{\mathrm{c}}=$ $0.24 R_{\mathrm{s}}$ and $\mu=5 \times 10^{11} \mathrm{~Pa}$ and viscosities consistent with the observed dissipation.

at $-3.5 \mathrm{Ga}$ in the case of fixed $\left|\operatorname{Im}\left(\tilde{k}_{2}\right)\right|$. As the semi-major axis decreases, due to the fluid response, $\left|\operatorname{Im}\left(\tilde{k}_{2 \mathrm{~s}}\right)\right|$ decreases (Fig. 2b) in the coupled model. Thus, $\mathrm{d} a / \mathrm{d} t$ becomes smaller as compared to the case with fixed $\left|\operatorname{Im}\left(\tilde{k}_{2 \mathrm{~s}}\right)\right|$, which results in the slow migration of Mimas.

Changes of semi-major axis at $R_{\mathrm{c}}=0.2 R_{\mathrm{S}}$ and $0.24 R_{\mathrm{S}}$ are shown in Fig. 4, respectively. Because the changing rate of the semi-major axis depends on $\left|\operatorname{Im}\left(\tilde{k}_{2 s}\right)\right|$, Mimas can avoid getting into the synchronous orbit regardless of core radius if the viscosity of Saturn's core is a lower boundary for the observed $k_{2 \mathrm{~s}} / Q_{\mathrm{s}}$. It is uncertain whether the viscosity of Saturn's solid core could be in the order of $10^{13} \mathrm{Pas}$. If $10^{14} \mathrm{~Pa}$ s of viscosity is required, Saturn should have a small core at around $0.2 R_{\mathrm{s}}$. Future studies with ab-initio calculations of the equation of state (EOS) and observations can constrain the interior structure of Saturn more precisely (e.g., Helled \& Guillot 2013; Miguel et al. 2016).

One caveat of our results is that, although $\sim 4 \times 10^{-5}$ of $\left|\operatorname{Im}\left(\tilde{k}_{2 s}\right)\right|$ is consistent with the observational values derived from each Saturnian satellite, it is smaller than the comprehensive evaluation at $\left|\operatorname{Im}\left(\tilde{k}_{2 \mathrm{~s}}\right)\right|=(1.59 \pm 0.74) \times 10^{-4}$ (Lainey et al. 2017). In the case of the simple two layer model, if $\left|\operatorname{Im}\left(\tilde{k}_{2 \mathrm{~s}}\right)\right| \sim 8 \times 10^{-5}$, Mimas gets into the synchronous orbit from the current position $\sim 3$ billion years ago. Calculations with more accurate core structure may solve this discrepancy, which will be addressed in future studies.

If we apply Eq. (1) to Enceladus, Tethys, and Dione, $\mathrm{d} a / \mathrm{d} t$ of Tethys with the current parameters could be larger than that of Mimas. Thus Tethys can expand faster than other satellites and further discussions about Tethys' orbital evolution may be required in future studies. The fast expansion is due to Tethys' greater mass (as compared to Mimas and Enceladus) and its relatively small semi-major axis (as compared to Dione). It is indicated that Tethys experienced large tidal heating like Enceladus in the past (Giese et al. 2007; Chen \& Nimmo 2008). Thus, the effect of dissipation in Tethys may not be negligible for its orbital evolution, which modifies Eq. (1) and requires detailed evolutions of the eccentricity and interior structure of Tethys.

\section{Conclusions}

Conventionally, the magnitude of Saturnian dissipation has been constrained from the orbital expansion of Mimas. Assuming a constant Love number, more than a few tens of thousands of Saturnian $Q$ is required for Mimas to stay outside of the surface of Saturn or the synchronous orbit 4.5 billion years ago (e.g., Goldreich \& Soter 1966; Gavrilov \& Zharkov 1977; Murray \& Dermott 2000; Meyer \& Wisdom 2007). However, the latest observations of Saturnian dissipation estimated at the orbital frequency of Enceladus, Tethys, and Dione imply that the Saturnian $Q$ is in the order of a few thousand, only 
(Lainey et al. 2017). We calculated the past semi-major axis of Mimas induced by tidal dissipation in Saturn's solid core assuming a Maxwell rheology and frequency dependence of $k_{2 \mathrm{~s}} / Q_{\mathrm{s}}$ $\left(\left|\operatorname{Im}\left(\tilde{k}_{2 s}\right)\right|\right)$. If the viscosity of Saturn's core is consistent with the lower boundary of the observed dissipation $\left(k_{2 \mathrm{~s}} / Q_{\mathrm{s}} \sim 4 \times 10^{-5}\right)$ (Lainey et al. 2017) and $\mu / \eta$ is larger than $2(\Omega-\omega) / 2 \pi$, due to the smaller tidal frequency in the past, Mimas can stay well outside the synchronous orbit even if it formed 4.5 billion years ago. The viscosity consistent with the observations and Mimas' expansion changes with the radius of the Saturnian core. If the core radius is $0.2 R_{\mathrm{s}}, 0.219 R_{\mathrm{s}}$ and $0.24 R_{\mathrm{s}}, \sim 10^{14} \mathrm{~Pa} \mathrm{~s}, \sim 5 \times 10^{13} \mathrm{~Pa} \mathrm{~s}$, and $\sim 2.5 \times 10^{13} \mathrm{~Pa}$ s of viscosities are required, respectively. In the case of these viscosity values, dissipation of Saturn is consistent with both the latest observational results and Mimas' orbital evolution. In this model, the assumption of a late formation discussed recently (Charnoz et al. 2011) is not required.

In this work, we assume that the solid core and the envelope are homogeneous. In addition, the envelope is assumed to be non-dissipative, and rigidity and viscosity of the core do not change with time. So far, whether a solid state core exists and its detailed structure are uncertain. We note that the Maxwell rheology used in our model would not be applicable if Saturn has a liquid core. Detailed interior structure models of Saturn and thermal evolution models can constrain the dissipation mechanisms more precisely, which should be addressed in future studies.

Acknowledgements. This work was supported by a JSPS Research Fellowship.

\section{References}

Anderson, J. D., \& Schubert, G. 2007, Science, 317, 1384

Auclair-Desrotour, P., Le Poncin-Lafitte, C., \& Mathis, S. 2014, A\&A, 561, L7 Chandrasekahr, S. 1969, Ellipsoidal Figures of Equilibrium. The Silliman Foundation Lectures (Yale Univ. Press)

Charnoz, S., Crida, A., Castillo-Rogez, J. C., et al. 2011, Icarus, 216, 535

Chen, E. M. A., \& Nimmo F. 2008, Geophys. Res. Lett., 35, L19203

Efroimsky, M., \& Lainey, V. 2007, J. Geophys. Res., 112, E12003

Fuller, J., Luan, J., \& Quataert, E. 2016, MNRAS, 458, 3867

Gavrilov, S. V., \& Zharkov, V. N. 1977, Icarus 37, 443

Giampieri, G., Dougherty, M. K., Smith, E. J., \& Russell, C. T. 2006, Nature, 441,62

Giese, B., Wagner, R., Neukum, G., Helfenstein, P., \& Thomas, P. C. 2007, Geophys. Res. Lett., 34, L21203

Goldreich, P., \& Soter, S. 1966, Icarus, 5, 375

Guenel, M., Mathis, S., \& Remus, F. 2014, A\&A, 566, L9

Helled, R., \& Guillot, T. 2013, ApJ, 767, 113

Jacobson, R. A., Antreasian, P. G., Bordi, J. J., et al. 2006, AJ, 132, 2520

Kaula, W. M. 1964, Rev. Geophys. Space Sci., 2, 661

Lainey, V., Karatekin, Ö, Desmars, J., et al. 2012, ApJ, 752, 14

Lainey, V., Jacobson, R. A., Tajeddine, R., et al. 2017, Icarus, 281, 286

MacDonald, G. 1964, Rev. Geophys. Space Phys., 2, 467

Meyer, J., \& Wisdom, J. 2007, Icarus, 188, 535

Miguel, Y., Guillot, T., \& Fayon, L. 2016, A\&A, 596, A114

Murray, C. D., \& Dermott, S. F. 2000, Solar system Dynamics (Cambridge University Press)

Ogilvie, G., \& Lin, D. N. C. 2004, ApJ, 610, 477

Porco, C. C., Helfenstein, P., Thomas, P. C., et al. 2006, Science, 311, 1393

Remus, F., Mathis, S., Zahn, J.-P., \& Lainey, V. 2012, A\&A, 541, A165

Remus, F., Mathis, S., Zahn, J.-P., \& Lainey, V. 2015, A\&A, 573, A23

Thomas, P. C., Burns, J. A., Helfenstein, P., et al. 2007, Icarus, 190, 573

Tobie, G., Mocquert, A., \& Sotin, C. 2005, Icarus, 177, 534

Wahl, S. M., Hubbard, W. B., \& Militzer, B. 2017, Icarus, 282, 183 